\title{
La enseñanza de Filosofía de la Biología en clave de ciencia o creencia. Algunas herramientas conceptuales para definir las preferencias docentes
}

\author{
Philosophy of Biology and the teaching of matters \\ affected by the relationship \\ between science and belief
}

\author{
ÁFRICA VILLANUEVA-FÉLEZ \\ Instituto de Gestión de la Innovación y el Conocimiento, INGENIO (CSIC-UPV) \\ VICENTE CLARAMONTE SANZ \\ Departamento de Lógica y Filosofía de la Ciencia de la Universitat de València \\ (España)
}

Recibido: 11-2-2013

Aprobado definitivamente: 23-2-2013

\section{RESUMEN}

Este artículo pretende obtener medidas cuantitativas sobre las actitudes manifestadas por los académicos hacia cuestiones relativas a la Filosofía de la Biología y el creacionismo, y a la docencia de la Biología en general; e igualmente, determinar la relación entre las actitudes o posiciones de dichos académicos sobre la Filosofía de la Biología y sus actitudes hacia la práctica docente. Al efecto, propone cinco factores cuantitativos. El artículo termina estableciendo ciertas correlaciones no causales entre algunos de estos cinco factores cuantitativos, para trazar un perfil significativo sobre las preferencias del profesorado en Filosofía de la Biología al impartir su enseñanza en cuestiones concernidas por la relación ciencia-creencia.

\section{PALABRAS CLAVE \\ FILOSOFÍA DE LA BIOLOGÍA, EDUCACIÓN, CREACIONISMO, DISEÑO INTELIGENTE, METODOLOGÍA}

C Contrastes. Revista Internacional de Filosofía: Suplemento 18 (2013), pp. 325-339. ISSN: 1136-9922

Departamento de Filosofía, Universidad de Málaga, Facultad de Filosofía y Letras

Campus de Teatinos, E-29071 Málaga (España) 


\begin{abstract}
This paper aims to develop quantitative measures of scholars' attitudes toward the Philosophy of Biology and creationism, along with general questions about the teaching of Biology. Moreover, the study analyses the relationships between these academics' orientations towards the Philosophy of Biology and their attitudes towards teaching practices. To that effect, we propose five quantitative factors. The results do establish certain correlations between these five factors. The paper ends by describing lecturers' preferences in relation to the Philosophy of Biology and their teaching of matters regarding the relationship between science and belief.
\end{abstract}

\title{
I. INTRODUCCIÓN
}

LAS PREGUNTAS QUE MOTIVARON EL PRESENTE ESTUDIO SERÍAN DOS. En primer lugar, ¿es posible obtener medidas cuantitativas robustas acerca de las actitudes manifestadas por los académicos hacia ciertos aspectos de la docencia sobre Biología en general, y en particular, sobre la docencia de la Filosofía de la Biología en aquellas cuestiones más sensibles desde el punto de vista de una cosmovisión creacionista? En segundo lugar, suponiendo una respuesta afirmativa a dicha pregunta, ¿es posible determinar la relación existente entre las posiciones de dichos académicos en relación a la Filosofía de la Biología y sus actitudes hacia la práctica docente?

Obtener respuestas basadas en un análisis empírico previo, consistentes y logradas mediante una metodología rigurosa, serían los principales objetivos perseguidos por el estudio expuesto a continuación.

\section{MARCO DE REFERENCIA}

Con frecuencia se pregunta a biólogos y profesores de Biología si creen en la evolución biológica. La pregunta sorprende. Tratándose de ciencia, la cuestión clave no parece estribar en creer o no creer, sino en disponer de evidencia científica favorable o carecer de ella. Pero además, obsérvese, nadie pregunta a un físico o a un químico, de modo análogo y respectivamente, si creen en la gravitación universal o en la electrólisis.

Ambas circunstancias indican que la idea de evolución suele ser vinculada a la creencia antes que al conocimiento científico. Al fin y al cabo, una explicación científica sobre el origen y la transformación de la vida alcanza valores asumidos por muchos individuos, porque condiciona su autopercepción como ser humano al debatir cuestiones como la aceptación o rechazo del ancestro común, la filogenia con los primates, el papel no finalista del azar y la selección natural en la transformación de las especies o la antigüedad del planeta Tierra.

Esta tensión ciencia-creencia a la hora de precisar la naturaleza epistemológica de las teorías empleadas en Biología sobre el origen y transformación 
de la vida y el estatus del ser humano entre los seres vivos, ha conducido a los partidarios de una cosmovisión creacionista a ensayar diversos discursos sincréticos entre la ciencia biológica y el creacionismo. El primero cristalizó con el creacionismo científico -o ciencia de la creación-, significado en cierta literatura publicada en Estados Unidos a principios de los 80 del siglo XX, y el más reciente florece una década más tarde con la inferencia del diseño inteligente. Sin embargo, ambos coincidían en su finalidad básica; introducir una concepción creacionista en las clases de Biología del sistema público de enseñanza.

Ante esta situación, quedan servidos algunos dilemas de conciencia y deontología profesional para el profesorado de Biología, quien advierte cómo, en su praxis docente cotidiana en el aula, su tarea puede verse condicionada por esa tensión ciencia-creencia. Especialmente si en un entorno receptivo a una cosmovisión creacionista pretende impartir teoría evolucionista o explicar nociones como las de ancestro común, filogenia entre el ser humano y otras especies, antigüedad de la Tierra, etc. Podría hacerse a sí mismo preguntas como, ¿hasta qué punto es compatible el creacionismo con una explicación científica en Biología?; o bien, ¿debo modificar la enseñanza de la ciencia en función de las creencias de mis estudiantes?; o también, ¿es admisible adaptar la exposición de las teorías impartidas en clase por respeto al pluralismo de sus creencias?; y otras similares.

En el marco de referencia recién descrito, este artículo pretende, a partir de un fundamento empírico y metodológico, ofrecer algunas herramientas conceptuales que faciliten definir el perfil de las actitudes manifestadas por el colectivo de profesores e investigadores en Filosofía de la Biología cuando su praxis recae sobre cuestiones concernidas por la apuntada tensión ciencia-creencia.

\section{Metodología}

\section{III.1. MUESTRA Y RECOLECCIÓN DE DATOS}

El estudio empírico empleado para el análisis se realizó entre una población de académicos universitarios iberoamericanos con interés en el área de Filosofía de la Biología. La muestra inicial se elaboró a partir de dos fuentes informativas. Por un lado, se recopilaron los nombres y correos de los participantes en el I Congreso de la Asociación Iberoamericana de Filosofía de la Biología (AIFIBI), celebrado del 28 al 30 de noviembre de 2012 en Valencia (España); con este procedimiento se identificaron 106 académicos. La segunda proviene de quienes se inscribieron en un Google Groups sobre Filosofía de la Biología desde un año antes de la celebración del I Congreso AIFIBI; sustrayendo aquellos académicos participantes en el susodicho Congreso, se obtuvieron los datos relativos a otros 58 individuos. Sumando ambas cifras, la muestra empleada está compuesta por 164 académicos universitarios. 
La recolección de datos se realizó mediante una encuesta on-line. En una primera versión, fue testada entre 4 académicos españoles y 2 latinoamericanos para identificar dificultades en la redacción e interpretación del cuestionario. Se consideró fundamental realizar este testeo en ambos ámbitos geográficos para abordar posibles problemas de tipo lingüístico. Con los comentarios recibidos en este primer testeo, se realizaron las oportunas modificaciones de la encuesta. Una segunda versión fue testada con 2 profesores de la Universitat de València y de la Universidad Nacional de Educación a Distancia (UNED).

El 23 de Diciembre de 2012 se realizó un primer envío de la versión final de la encuesta a los 164 académicos indicados. Se realizó un segundo el 22 de enero del 2013, para incrementar las respuestas obtenidas en principio (Dillman, 2007). Se recibieron un total de 69 cuestionarios, de los cuales 43 provenían de los asistentes al I Congreso AIFIBI y 26 de los miembros del citado Google Groups. El índice de respuestas final constituye el $42 \%$ del total de la muestra inicial; $41 \%$ de la submuestra del Congreso y $45 \%$ de la submuestra del grupo de discusión. Según Dillman (2007), los índices de respuesta de los cuestionarios on-line superiores al $35 \%$ son aceptables.

\section{III.2. MEDIDAS DE LAS VARIABLES}

La encuesta fue diseñada mediante unos enunciados o ítems con los cuales los encuestados podían expresar su grado de acuerdo. Se utilizó una escala tipo Likert de 10 valores, donde 1 equivale a «Totalmente en desacuerdo» y 10 equivale a «Totalmente de acuerdo». En el cuestionario, los enunciados se presentaron en tres bloques diferentes. El primer y segundo bloque hacían referencia a cuestiones relativas a Filosofía de la Biología y creacionismo; el tercero fue diseñado sobre cuestiones docentes.

La tabla 1 muestra los enunciados o ítems empleados en el cuestionario, así como la media aritmética y la desviación típica obtenidas para cada uno.

\begin{tabular}{lcc}
\hline & Media & $\begin{array}{c}\text { Desviación } \\
\text { típica }\end{array}$ \\
\hline FILOSOFÍA DE LA BIOLOGÍA / CREACIONISMO & & \\
\hline $\begin{array}{l}\text { FB-Ítem 1 En Biología, la teoría de la evolución es una } \\
\text { concepción científica entre otras alternativas }\end{array}$ & 2,45 & 2,143 \\
$\begin{array}{l}\text { FB-Ítem 2 En Biología, la evolución es una teoría entre } \\
\text { otras teorías científicas }\end{array}$ & 2,55 & 2,644 \\
$\begin{array}{l}\text { FB-Ítem 3 En Biología, la evolución y el diseño } \\
\text { inteligente son teorías científicas alternativas }\end{array}$ & 1,41 & 1,390 \\
$\begin{array}{l}\text { FB-Ítem } 4 \text { En Biología, la teoría de la evolución es la } \\
\text { concepción científica principal }\end{array}$ & 7,87 & 2,493
\end{tabular}


FB-Ítem 5 Nada tiene sentido en Biología salvo bajo la luz de la evolución

FB-Ítem 6 Es posible fundamentar científicamente una explicación teística evolutiva sobre el origen de las especies

FB-Ítem 7 En Biología, la noción de teleología o finalidad es un concepto teórico imprescindible para desarrollar explicaciones científicas

FB-Ítem 8 Las carencias de la teoría evolucionista confirman el diseño inteligente como teoría científica

\section{CUESTIONES DOCENTES}

CD-Ítem 1 Es deseable que una docencia pluralista ampare la posibilidad de fundamentar científicamente las creencias del alumnado

CD-Ítem 2 Impartir teorías científicas alternativas, como la evolucionista y el diseño inteligente, contribuye a

fomentar el espíritu crítico en el alumnado

CD-Ítem 3 Entre varias teorías científicas, es preferible impartir aquélla que mejor concuerde con las creencias mayoritarias del alumnado

Tabla 1: Descriptivos de los ítems utilizados en la encuesta

En esta tabla puede observarse, a través de las medias obtenidas, que los enunciados en que se acepta la existencia de teorías alternativas a la evolución (FB ítems 1, 2 y 3) y el creacionismo como explicación científica (FB ítems 6, 7 y 8) tienen poca aceptación entre los encuestados. En cambio, en sentido contrario, el grado de acuerdo de aquellos enunciados que expresan conformidad con la teoría de la evolución como explicación dominante en Biología (FB ítems 4 y 5) es bastante elevado, con unas medias superiores a 7,5. Los cuatro enunciados empleados para determinar actitudes sobre cuestiones docentes muestran medias bastante bajas, y por tanto, poca aceptación entre los encuestados.

\section{III.3. TÉCNICAS DE ANÁLISIS}

El primer objetivo del estudio plantea si pueden construirse medidas cuantitativas robustas sobre las actitudes de los académicos hacia cuestiones relativas a Filosofía de la Biología y creacionismo, así como hacia cuestiones docentes en Biología. Para ello, se han empleado análisis factoriales exploratorios. Esta técnica multivariante permite observar la estructura subyacente de una serie de variables y reducir el mayor número de ellas -en este estudio, enunciados o 
ítems-al menor número de factores (Hair, Anderson et al., 1998; Uriel Jiménez y Aldás Manzano, 2005). El método de extracción utilizado fue el basado en componentes principales.

Para dotar de solidez a los análisis efectuados y validar los resultados obtenidos, se procedió así (Uriel Jiménez y Aldás Manzano, 2005). Primero se segmentó la muestra final entre los participantes al I Congreso AIFIBI y los miembros del grupo virtual de discusión; después se efectuaron los análisis de componentes principales en cada una de estas submuestras. Este proceder ha confirmado que los resultados obtenidos, tanto en cada una de las submuestras como en el total, no difieren significativamente.

El segundo objetivo del estudio plantea determinar la relación entre las actitudes de los académicos en relación a la Filosofía de la Biología y sus tendencias hacia la práctica docente, para lo cual se han empleado correlaciones bivariadas de Pearson.

\section{RESULTADOS Y DISCUSIÓN}

Para quienes no estén familiarizados con los diagramas sobre carga factorial (tablas 2 y 3), incluimos a continuación unos apuntes que facilitarán su lectura. Con sus columnas de cifras, la tabla 2 no muestra, como podría parecer, el grado de aceptación de los encuestados sobre cada ítem, sino el peso específico -de ahí «carga factorial»- aportado por cada ítem a la configuración y consistencia de cada factor. Por ejemplo, el factor FB-F1 (Grado de compatibilidad de la teoría evolucionista con otras explicaciones científicas en Biología) se ha configurado agrupando 3 ítems (1, 2 y 3 ); pues bien, las cifras $0,868,0,859$ y 0,686 (columna «Total») indican cuánto cargan tales ítems en la configuración del factor FB-F1, es decir, en qué medida contribuyen a su consistencia. Por ello, la comprensión de la tabla se facilita al leer por columnas -en vertical- y asociando las cifras con tipografía negrita.

Y desde el punto de vista metodológico, los factores obtenidos con la técnica empleada para elaborar las tablas 2 y 3 , son nuevas variables calculadas como combinación lineal de los valores de cada ítem en cada uno de los encuestados por su carga factorial. Por ejemplo: FB-F1 $1_{i \text { del Total }}=0,868 \times$ FB-Ítem $1_{i}+0,859 \times$ $F B$-Ítem $2_{i}+0,686 \times$ x FB-Ítem $3_{i}-0,052 \times F B$-Item $4_{i}-0,270 \times$ x FB-Ítem $5_{i}+0,097$ x FB-Ítem $6_{i}+0,250 \times$ x FB-Ítem $7_{i}-0,210 \times$ FB-Ítem $8_{i}$.

El resto de factores son calculados análogamente con sus respectivas cargas factoriales. A través de la interpretación de los factores resultantes, éstos se dotan de etiquetas que expresan con una sola idea la variable medida por los ítems agrupados gracias a la compatibilidad de sus resultados en cifras.

La tabla 2 muestra los factores y sus cargas factoriales, obtenidos a partir del análisis de los componentes principales efectuado sobre los ítems contenidos en los bloques sobre Filosofía de la Biología en relación con el creacionismo, 


\begin{tabular}{|c|c|c|c|c|c|c|c|c|c|}
\hline \multirow[b]{2}{*}{ ÍTEMS } & \multicolumn{3}{|c|}{$\begin{array}{l}\text { FB-F1: Grado de } \\
\text { compatibilidad de la } \\
\text { teoria evolucionista con } \\
\text { otras explicaciones } \\
\text { cientificas en Biologia }\end{array}$} & \multicolumn{3}{|c|}{$\begin{array}{c}\text { FB-F2: Grado de } \\
\text { aceptación de la teoría } \\
\text { evolucionista como } \\
\text { explicación dominante en } \\
\text { Biología }\end{array}$} & \multicolumn{3}{|c|}{$\begin{array}{l}\text { FB-F3: Grado de } \\
\text { compatibilidad del } \\
\text { creacionismo con una } \\
\text { explicación cientifica en } \\
\text { Biologia }\end{array}$} \\
\hline & Total & AIFIBI & $\begin{array}{l}\text { Grupo } \\
\text { Virtual }\end{array}$ & Total & AIFIBI & $\begin{array}{l}\text { Grupo } \\
\text { Virtual }\end{array}$ & Total & AIFIBI & $\begin{array}{l}\text { Grupo } \\
\text { Virtual }\end{array}$ \\
\hline $\begin{array}{l}\text { FB-Ítem } 1 \text { En Biologia, la teoría de la } \\
\text { evolución es una concepción científica } \\
\text { entre otras alternativas }\end{array}$ & 0,868 & 0,842 & 0,921 & $-0,110$ & $-0,034$ & $-0,266$ & $-0,070$ & $-0,015$ & 0,134 \\
\hline $\begin{array}{l}\text { FB-Ítem } 2 \text { En Biologia, la evolución es } \\
\text { una teoria entre otras teorias cientificas }\end{array}$ & 0,859 & 0,845 & 0,865 & $-0,086$ & $-0,293$ & 0,150 & 0,050 & 0,050 & $-0,147$ \\
\hline $\begin{array}{l}\text { FB-Ítem } 3 \text { En Biologia, la evolución y } \\
\text { el diseño inteligente son teorias } \\
\text { cientificas alternativas }\end{array}$ & 0,686 & 0,692 & 0,725 & $-0,139$ & $-0,067$ & $-0,495$ & 0,120 & 0,101 & 0,092 \\
\hline $\begin{array}{l}\text { FB-Ítem } 4 \text { En Biologia, la teoria de la } \\
\text { evolución es la concepción cientifica } \\
\text { principal }\end{array}$ & $-0,052$ & $-0,226$ & 0,027 & 0,911 & 0,887 & 0,900 & $-0,013$ & $-0,158$ & $-0,052$ \\
\hline $\begin{array}{l}\text { FB-Ítem } 5 \text { Nada tiene sentido en } \\
\text { Biologia salvo bajo la luz de la } \\
\text { evolución }\end{array}$ & $-0,270$ & $-0,072$ & $-0,296$ & 0,864 & 0,867 & 0,873 & $-0,118$ & 0,035 & 0,007 \\
\hline $\begin{array}{l}\text { FB-Ítem } 6 \text { Es posible fundamentar } \\
\text { cientificamente una explicación teistica } \\
\text { evolutiva sobre el origen de las especies }\end{array}$ & 0,097 & 0,105 & 0,035 & 0,139 & 0,178 & $-0,097$ & 0,807 & 0,848 & 0,869 \\
\hline $\begin{array}{l}\text { FB-Ítem } 7 \text { En Biologia, la noción de } \\
\text { teleologia o finalidad es un concepto } \\
\text { teórico imprescindible para desarrollar } \\
\text { explicaciones científicas }\end{array}$ & 0,250 & 0,336 & 0,034 & $-0,151$ & $-0,167$ & 0,166 & 0,746 & 0,693 & 0,731 \\
\hline $\begin{array}{l}\text { FB-Ítem } 8 \text { Las carencias de la teoria } \\
\text { evolucionista confirman el diseño } \\
\text { inteligente como teoria cientifica }\end{array}$ & $-0,210$ & $-0,317$ & $-0,034$ & $-0,143$ & $-0,226$ & $-0,122$ & 0,614 & 0,593 & 0,616 \\
\hline \multicolumn{10}{|c|}{ KMO: Total $=0,671 ;$ AIFIBI $=0,520 ;$ Grupo virtual $=0,504$} \\
\hline \multicolumn{10}{|c|}{ Varianza Explicada: Total $=68 \% ;$ AIFIBI $=69 \% ;$ Grupo virtual $=74 \%$} \\
\hline Método de rotación: Normalización Varin & con Kais & & & & & & & & \\
\hline
\end{tabular}

Tabla 2: Factores resultantes sobre Filosofía de la Biología/Creacionismo

Esta tabla 2 muestra cómo el primer factor FB-F1 se construye por agrupación de los enunciados FB ítems 1, 2 y 3, los cuales, considerados en conjunto, según este estudio permiten cuantificar el grado de compatibilidad entre la teoría evolucionista y otras explicaciones científicas en Biología. Entendemos que ello es así por estos motivos. Responder a favor de los FB-Ítem 1 y FBÍtem 2 , ambos parecidos, equivale mostrarse proclive a considerar que la teoría de la evolución es compatible con otras explicaciones científicas alternativas. El enunciado FB-Ítem 3 especifica esta misma idea cuando la alternativa a la teoría de la evolución se concreta en el diseño inteligente, si acaso este discurso pudiera considerarse una teoría científica; por tanto, mostrarse favorable a dicho ítem equivale a sostener la compatibilidad de la teoría evolucionista con otras explicaciones, en este caso, con el diseño inteligente. 
El factor FB-F2 se construye agrupando los enunciados FB ítems 4 y 5 . A partir de la tabla 2 antes expuesta, según este estudio ambos permiten cuantificar el grado de aceptación de la teoría evolucionista como explicación dominante en Biología. En este caso, los enunciados son tan explícitos que creemos superfluo justificar adicionalmente que su agrupación equivale a otorgar a la teoría evolucionista el estatus epistemológico de teoría dominante en Biología. El primero casi traduce literalmente el contenido cuantificado por el factor; y en cuanto a la célebre cita de Dobzhansky, resulta difícil discutir la equivalencia entre aceptar que el conocimiento científico en Biología solo tiene sentido bajo el prisma teórico del evolucionismo y aceptar que, en dicha área científica, la teoría evolucionista es la explicación dominante. Al menos, nosotros no hallamos argumentos racionales para discutirla.

Por último, el tercer factor FB-F3 se construye agrupando los enunciados FB ítems 6,7 y 8 . Este estudio considera que su conjunto triádico permite cuantificar el grado de compatibilidad del creacionismo con una explicación científica en Biología. Es decir, este factor mediría hasta qué punto las respuestas tienden a admitir la posibilidad de articular en Biología una teoría científica aceptora y no contradictora de los postulados principales del creacionismo. El FB-Ítem 6 apunta con claridad hacia ese sentido, pues equivale a conciliar una explicación científica con la intervención de un dios personal y providente creador del mundo - dogma irrenunciable para el creacionismo- en la génesis de los seres vivos. La justificación del FB-Ítem 7 quizás requiera una explicación más detallada. Casi unánimemente, las cosmovisiones creacionistas asumen la teleología o el finalismo como un ingrediente o una consecuencia ineludible en Biología: el origen y diversidad de los seres vivos responden a la finalidad prevista para ellos por el ser sobrenatural creador. Baste recordar el argumento teleológico para demostrar la existencia de Dios debido a Tomás de Aquino ${ }^{1}$ y el argumento del relojero propuesto por William Paley. ${ }^{2}$ Sin embargo, no es menos cierto que autores como Ernst Mayr y Francisco Ayala ${ }^{3}$ han reintroducido con pleno rigor las causas teleonómicas en el marco de una investigación científica empíricamente verificable. Ahora bien, su tesis en esta sede consiste en negar que toda explicación teleológica debería ser excluida de la teoría evolucionista, pero no

1 Sintetizado, su silogismo sería básicamente este: El ser sin entendimiento obra, no obstante serlo, por un fin; el ser sin entendimiento sólo puede cumplir su fin si es dirigido; por tanto, debe existir un ser inteligente que dirija al ser sin entendimiento y dicho ser rector es Dios.

2 Ídem: Cuando observamos un reloj y cómo sus diversas partes están perfectamente ensambladas con un propósito, pensamos inetivablemente que debe haber tenido un relojero fabricante. De modo análogo, al observar cómo los seres vivos están perfectamente integrados en la naturaleza con un propósito, inferimos que debe haber tenido un creador a quien llamamos Dios.

3 Ayala, Dobzhansky et al. (1983), pp. 494-499. 
establecen una defensa a ultranza del finalismo como ingrediente inevitable en una teoría científico-biológica. Aquí aparece el elemento diferencial entre su posición y el enunciado FB-Ítem 7, en el calificativo «imprescindible», para desarrollar una teoría científica, aplicado a la noción de teleología. Dicha clave trazaría el perfil creacionista de las respuestas favorables, pues la imprescindibilidad del finalismo en toda explicación científico-biológica solo puede defenderse desde una concepción creacionista al estilo de Aquino, Paley o la inferencia del diseño inteligente, mientras el pensamiento evolucionista acepta sin problemas el azar-es decir, la negación del finalismo- en una fuerza evolutiva como la deriva genética, así como en otros elementos del tejido conceptual evolucionista. Ello otorgaría fuerza dirimente al FB Ítem 7 para señalar las respuestas favorables a la compatibilidad entre el creacionismo -el evolucionismo no puede defender la imprescindibilidad del finalismo-y una explicación científica, y sin contravenir posturas como las de Mayr y Ayala, para quienes ciertas explicaciones teleológicas sí serían admisibles en la órbita de la teoría evolucionista. Por último, el enunciado del FB-Ítem 8 no hace sino reformular el argumento negativo del diseño inteligente: «Todo lo que niega la teoría evolutiva confirma el diseño inteligente». Siendo el diseño inteligente la última variante del creacionismo científico, resulta evidente que mostrarse favorable a su argumento negativo equivale a aceptar la compatibilidad entre el creacionismo y una explicación científica en Biología; tal y como sostenemos al categorizar el factor FB-F3 justificado en este párrafo.

De forma análoga a lo expuesto antes, se han analizado los enunciados sobre cuestiones docentes. La tabla 3 muestra los factores y cargas factoriales resultantes de las actitudes de los encuestados sobre tales cuestiones. Como puede observarse, se han utilizado 4 enunciados agrupados en torno a 2 factores, CD-F1 y CD-F2. Las pruebas de U de Mann-Whitney realizadas con ambos también señalan la inexistencia de diferencias significativas entre las dos submuestras, validando así los resultados presentados en la tabla 3 .

\begin{tabular}{|c|c|c|c|c|c|c|}
\hline \multirow[b]{2}{*}{ ÍTEMS } & \multicolumn{3}{|c|}{$\begin{array}{c}\text { CD-F1: Grado de } \\
\text { adaptabilidad del currículo } \\
\text { por motivos de pluralismo }\end{array}$} & \multicolumn{3}{|c|}{$\begin{array}{c}\text { CD-F2: Grado de } \\
\text { alterabilidad de la docencia } \\
\text { en función de las creencias } \\
\text { del discente }\end{array}$} \\
\hline & Total & AIFIBI & $\begin{array}{l}\text { Grupo } \\
\text { Virtual }\end{array}$ & Total & AIFIBI & $\begin{array}{l}\text { Grupo } \\
\text { Virtual } \\
\end{array}$ \\
\hline $\begin{array}{l}\text { CD-Ítem } 1 \text { Es deseable que } \\
\text { una docencia pluralista ampare } \\
\text { la posibilidad de fundamentar } \\
\text { científicamente las creencias del } \\
\text { alumnado }\end{array}$ & 0,905 & 0,911 & 0,918 & $-0,045$ & $-0,065$ & $-0,053$ \\
\hline
\end{tabular}




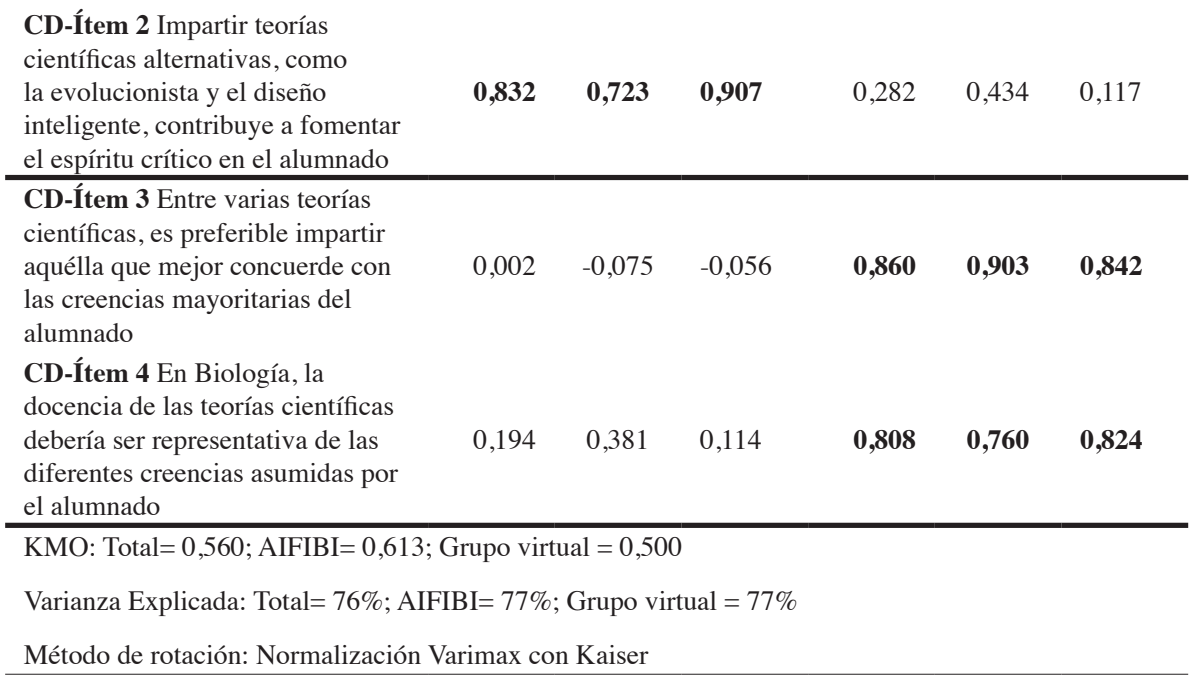

Tabla 3: Factores resultantes sobre cuestiones docentes

En esta tabla 3, los enunciados CD ítems 1 y 2 han sido agrupados en el factor CD-F1, con el cual proponemos medir el grado de adaptabilidad del currículo por motivos de pluralismo. El CD-Ítem 1 sugiere que sus partidarios tenderán a evitar la indeseable situación de un currículo que niegue al alumnado la posibilidad de estudiar el fundamento científico de sus creencias.

En cuanto al CD-Ítem 2, quien se muestre favorable al mismo prefiere buscar con su docencia la formación del espíritu crítico, cuya premisa es un marco de discusión pluralista; cuando el estado del conocimiento científico señale que solo una teoría debe integrar el currículo, seguir su propia orientación docente le conducirá a admitir teorías alternativas, especialmente si el alumnado lo reclama, y así propenderá a modificar el currículo académico.

El factor CD-F2 se propone para medir el grado de alterabilidad de la docencia en función de las creencias del discente y agrupa los enunciados CD ítems 3 y 4. La subsunción bajo este factor del CD-Ítem 3 se justifica por entender que, quien se muestre favorable, también optará por adaptar el contenido de sus clases según el criterio de la teoría científica más acorde a las creencias preferidas por la mayoría de estudiantes, única forma de impartir docencia con tal criterio cuando la teoría preferida por ellos sea incompatible con la designada por el criterio habitualmente empleado para elaborar los currículos de ciencia, es decir, la sintonía con el estado coetáneo del conocimiento científico y su preferencia mayoritaria entre la comunidad científica. Y la inclusión CD-Ítem 4 se explica por motivos análogos: quien se muestre favorable a este enunciado, tenderá a ajustar su exposición de las teorías científicas a las creencias de sus estudiantes. 
Por tanto, cuando la teoría científica a exponer no sea representativa de éstas, si de verdad prefiere aplicar el criterio de la representatividad expuesto en el enunciado, deberá alterar su docencia en función de las creencias del discente.

En definitiva, recapitulando los párrafos anteriores, puede afirmarse que las tablas 2 y 7 confirman la consecución del primer objetivo del estudio.

El segundo objetivo persigue responder a dos cuestiones: comprobar si existe o no una relación significativa entre los posicionamientos de los encuestados ante cuestiones de Filosofía de la Biología/creacionismo y sus actitudes docentes, y determinar si las relaciones detectadas son directas o inversas. Para ello, como se ha avanzado antes, se han calculado los coeficientes de correlación de Pearson. Estos coeficientes miden el grado de relación entre dos pares de variables, en este estudio, los factores antes obtenidos.

La tabla 4 muestra las correlaciones entre factores. Sorprende que el factor (4) FB-F2 (Grado de aceptación de la teoría evolucionista como explicación dominante en Biología) no se correlaciona significativamente con ningún factor referente a actitudes docentes; es decir, aceptar la teoría evolucionista como explicación dominante no se corresponde con actitudes proclives a adaptar el currículum docente o alterar la docencia para acercarla a las creencias religiosas del alumnado.

\begin{tabular}{|c|c|c|c|c|c|}
\hline & (1) & (2) & (3) & (4) & (5) \\
\hline $\begin{array}{l}\text { (1) CD-F1 Grado de } \\
\text { adaptabilidad del currículo } \\
\text { por motivos de pluralismo }\end{array}$ & 1 & 0,000 & $0,277^{*}$ & 0,058 & $-0,073$ \\
\hline $\begin{array}{l}\text { (2) CD-F2 Grado de } \\
\text { alterabilidad de la docencia } \\
\text { en función de las creencias del } \\
\text { discente }\end{array}$ & 0,000 & 1 & $0,294^{*}$ & $-0,126$ & $0,503^{* *}$ \\
\hline $\begin{array}{l}\text { (3) FB-F1 Grado de } \\
\text { compatibilidad de la teoría } \\
\text { evolucionista con otras } \\
\text { explicaciones científicas en } \\
\text { Biología }\end{array}$ & $0,277^{*}$ & $0,294^{*}$ & 1 & 0,000 & 0,000 \\
\hline $\begin{array}{l}\text { (4) FB-F2 Grado de } \\
\text { aceptación de la teoría } \\
\text { evolucionista como } \\
\text { explicación dominante en } \\
\text { Biología }\end{array}$ & 0,058 & $-0,126$ & 0,000 & 1 & 0,000 \\
\hline $\begin{array}{l}\text { (5) FB-F3 Grado de } \\
\text { compatibilidad del } \\
\text { creacionismo con una } \\
\text { explicación científica en } \\
\text { Biología }\end{array}$ & $-0,073$ & $0,503^{* *}$ & 0,000 & 0,000 & 1 \\
\hline$* \mathrm{p}<0.01 ; * \mathrm{p}<0.05$ & & & & & \\
\hline
\end{tabular}

Tabla 4: Correlaciones bivariadas entre los factores 
Sin embargo, el factor (3) FB-F1 (Grado de compatibilidad de la teoría evolucionista con otras explicaciones científicas en Biología), muestra una relación significativa y directa o positiva con los dos factores referentes a cuestiones docentes. Es decir, quienes admitan la existencia de teorías científicas compatibles con el evolucionismo, tenderán a adaptar la docencia por cuestiones pluralistas, pero también a alterar la docencia por cuestiones dogmáticas; y viceversa.

Por último, el factor (5) FB-F3 (Grado de compatibilidad del creacionismo con una explicación científica en Biología) se correlaciona muy fuerte y positivamente con el factor que mide el grado de alterabilidad de la docencia. Es decir, a mayor aceptación del creacionismo como explicación científica, mayor grado de alterabilidad de la docencia en función de las creencias de los discentes.

En definitiva, puede afirmarse que la tabla 4 confirma el segundo objetivo de este estudio.

Por último, la tabla 5 presenta el grado de aceptación de los encuestados sobre los factores obtenidos.

\begin{tabular}{|c|c|c|c|}
\hline & Total & AIFIBI & $\begin{array}{l}\text { Grupo } \\
\text { Virtual }\end{array}$ \\
\hline & $\begin{array}{c}\% \text { muestra a } \\
\text { favor }\end{array}$ & $\begin{array}{c}\% \text { muestra a } \\
\text { favor }\end{array}$ & $\begin{array}{c}\% \text { muestra a } \\
\text { favor }\end{array}$ \\
\hline \multicolumn{4}{|l|}{$\begin{array}{l}\text { FILOSOFIA DE LA BIOLOGÍA / } \\
\text { CREACIONISMO }\end{array}$} \\
\hline $\begin{array}{l}\text { FB-F1 Grado de compatibilidad } \\
\text { de la teoría evolucionista con otras } \\
\text { explicaciones científicas en Biología }\end{array}$ & $34,5 \%$ & $33,3 \%$ & $36,8 \%$ \\
\hline $\begin{array}{l}\text { FB-F2 Grado de aceptación de la } \\
\text { teoría evolucionista como explicación } \\
\text { dominante en Biología }\end{array}$ & $63,6 \%$ & $55,6 \%$ & $78,9 \%$ \\
\hline $\begin{array}{l}\text { FB-F3 Grado de compatibilidad del } \\
\text { creacionismo con una explicación } \\
\text { científica en Biología }\end{array}$ & $34,5 \%$ & $38,9 \%$ & $26,3 \%$ \\
\hline $\mathrm{N}$ & 55 & 36 & 19 \\
\hline Perdidos & 14 & 7 & 7 \\
\hline \multicolumn{4}{|l|}{ CUESTIONES DOCENTES } \\
\hline $\begin{array}{l}\text { CD-F1 Grado de adaptabilidad del } \\
\text { currículo por motivos de pluralismo }\end{array}$ & $32,1 \%$ & $34,3 \%$ & $28,6 \%$ \\
\hline $\begin{array}{l}\text { CD-F2 Grado de alterabilidad de la } \\
\text { docencia en función de las creencias } \\
\text { del discente }\end{array}$ & $19,6 \%$ & $20 \%$ & $19 \%$ \\
\hline $\mathrm{N}$ & 56 & 35 & 21 \\
\hline Perdidos & 13 & 8 & 5 \\
\hline
\end{tabular}

Tabla 5: Distribución de la muestra entre los distintos factores 
Esta tabla 5 requiere pocas explicaciones. En términos generales, podrían establecerse las siguientes afirmaciones sobre las preferencias de los encuestados: la mayoría es proclive a la teoría evolucionista como explicación dominante; solo $1 / 3$ tiende a aceptar que dicha teoría sea compatible con otras teorías científicas y que el creacionismo sea compatible con una explicación científica; apenas $1 / 3$ tenderían a adaptar el currículo por motivos de pluralismo y, por último, apenas 1/5 muestran tendencia a alterar su docencia en función de las creencias del discente.

\section{CONCLUSIONES}

Los objetivos de este estudio se han cumplido. Por un lado, se han obtenido medidas robustas sobre conceptos alusivos a perspectivas sobre Filosofía de la Biología y a actitudes sobre su docencia; por otro, se ha determinado la relación existente entre dichas perspectivas y actitudes (tabla 5).

El acuerdo mayoritario entre las preferencias de los encuestados gravita (tabla 5) sobre el éxito de la teoría evolucionista como explicación científica dominante en Biología (64\%) y sobre no alterar la docencia en función de las creencias del discente (80\%). En este último caso, y en sentido contrario, sorprende que un $20 \%$ del personal académico encuestado se manifieste proclive a alterar su docencia sobre teorías científicas, aunque carecemos de verificación acerca de que, más allá de esta predisposición, tales actitudes se apliquen en la práctica. Esta tendencia a la alterabilidad de la docencia de las teorías científicas se nutre (tabla 4) de considerar compatible la teoría evolucionista con otras explicaciones científicas y, sobre todo, de aceptar la compatibilidad entre el creacionismo y una explicación científica válida. Pero además, aunque la correlación no sea significativa, resulta interesante observar que, a mayor grado de aceptación de la teoría evolucionista, menor es la propensión a alterar el currículo.

No obstante lo anterior, la tendencia proclive a la adaptabilidad del currículo por motivos de pluralismo no depende de aceptar al creacionismo como una explicación científica en Biología, sino más bien, del ánimo de fomentar el espíritu crítico entre el estudiantado. Es decir, cuanto mayor es la apuesta por el creacionismo, mayor es la tendencia a alterar -no a adaptar- el currículo; de hecho, la relación entre adaptabilidad del currículo y creacionismo es negativa, aunque no sea significativa.

Los autores de este estudio son conscientes de sus limitaciones, especialmente en cuanto al número de casos analizados, lo cual a su vez impide realizar otros tipos de análisis, como un factorial confirmatorio, y también en cuanto a la herramienta de recogida de datos -encuesta-, pues los ítems utilizados podrían haberse formulado con mayor precisión. Lo proponen solo como primera aproximación al estudio de las preferencias de los académicos interesados en Filosofía de Biología, abierta a su perfeccionamiento futuro a través de sucesivas 
investigaciones metodológicamente refinadas y de las contribuciones críticas del propio colectivo encuestado.

\section{Agradecimientos}

Siendo muchas para citarlas todas por sus nombres y apellidos, no obstante, de modo genérico pero expreso quisiéramos agradecer a las personas asistentes al I Congreso de la Asociación Iberoamericana de la Filosofía de la Biología, así como a las integrantes del Google Group Filósofos de la Biología, su colaboración al responder la encuesta on-line que ha permitido fundamentar empíricamente este estudio. Y muy especialmente, a las Doctoras y Doctores Alcolea Banegas, Casaban Moya, Parreño Rabadán, Peretó Magraner, Saborido Alejandro, Tamayo Hurtado y Valor Abad, su ayuda de diversa índole al configurar la herramienta metodológica empleada en el mismo. Sin el apoyo de todas, citadas de modo genérico o específico, este artículo no hubiera podido elaborarse con resultados satisfactorios.

\section{REFERENCIAS BIBLIOGRÁFICAS}

AYALA, DOBZHANSKY et al. (1983), Evolución. Barcelona: Omega.

DILLMAN, D. A. (2007). Mail and internet surveys: The tailored design method. Hoboken, New Jersey: John Wiley \& Sons, Inc.

HAIR, J. , ANDERSON, R. et al. (1998). Multivariate Data Analysis. Upper Saddle River, NJ: Prentice-Hall International, Inc.

URIEL E. y ALDÁS, J. (2005). Análisis multivariante aplicado. Madrid: Thomson Editores Spain.

África Villanueva-Félez es investigadora del Instituto de Gestión de la Innovación y el Conocimiento, INGENIO (CSIC-UPV).

Líneas de investigación:

Sociología del conocimiento, redes sociales, relaciones universidad-sociedad, transferencia de conocimiento, evaluación de política científica, educación.

Publicaciones recientes:

MOLAS-GALLART, J.;VILLANUEVA-FELEZ,A.; FERNÁNDEZ-DE-LUCIO, I. (2011), Análisis de la evolución de los parques científicos españoles. Fundación Cotec para la Innovación. Tecnológica España, ISBN: 978-8-4929-3313-6 
VILLANUEVA-FÉLEZ,A.; BEKKERS,R.; MOLAS-GALLART,J.(2010), «University-industry relationships and the role of the individual: networking ties and the diversity of knowledge transfer». Industry and High Education, 24 (3): 203-210.

Dirección electrónica: africa.villanueva@ingenio.upv.es

Vicente Claramonte Sanz es profesor del Departamento de Lógica y Filosofía de la Ciencia de la Universitat de València.

Líneas de investigación:

Filosofía de la Ciencia, Filosofía de la Biología, ciencia y religión, evolucionismo y creacionismo, diseño inteligente.

Publicaciones recientes:

CLARAMONTE, V. (2011), Diseño inteligente: la pseudociencia del siglo XXI, Lambert Academic Publishing: Saarbrücken, ISBN 978-3-8443-3627-6

TAMAYO, M. y CLARAMONTE, V. (2011), Evolución y creación. Los argumentos antievolucionistas ante las evidencias científicas, Lambert Academic Publishing: Saarbrücken, ISBN 978-3-8454-9103-5

Dirección electrónica: vicente.claramonte@uv.es 